\title{
Design and Analysis of Robust Track-Following Controllers for Dual-Stage Servo Systems with an Instrumented Suspension
}

\author{
Xinghui Huang, Roberto Horowitz, and Yunfeng Li
}

\begin{abstract}
This paper discusses the design and analysis of two robust track-following controllers for a dual-stage servo system containing a MEMS microactuator and an instrumented suspension in hard disk drives. The first controller is designed using the so-called PQ method, which is a kind of sequential single-input/single-output (SISO) frequency shaping design techniques. Actuation interference is explicitly addressed in this design. The second controller is designed using a multi-objective optimization technique via linear matrix inequalities (LMIs). Stability robustness to plant uncertainty can be explicitly considered by imposing some $H_{\infty}$ norm bounds. In both cases, compensation of airflow-excited suspension vibration is implemented first by using the strain sensor output from the instrumented suspension. Detailed analysis and comparison are carried out on system dynamics, robust stability, robust performance, and implementation complexity.
\end{abstract}

\section{INTRODUCTION}

There is a continuing demand for higher areal densities in computer hard disk drives. To maintain a high level of trackfollowing performance at a reduced track pitch, a trackfollowing servo system must be able to compensate for the track misregistration (TMR) caused by various factors such as track runout, disk flutter, windage disturbance, and external shock and vibrations. This can be effectively accomplished by increasing the servo bandwidth of the system. Dual-stage actuator (DSA) systems have thus been proposed and intensively studied as a prospective solution to boosting servo bandwidth, which in conventional singlestage actuators is otherwise limited mainly by the presence of suspension resonance modes. According to the location of a microactuator relative to the VCM, DSA systems are classified into three major types: actuated suspension [1][2], actuated slider [3][4] and actuated read/write head [5], all havng specific advantages and disadvantages.

In this paper, we discuss the design of robust trackfollowing controllers for a dual-stage actuated-slider servo system. The dual-stage system has an electrostatic MEMS microactuator (MA) between the suspension tip and the slider. The MA can generate translational relative motion of the slider with respect to the suspension tip, and this relative motion can be sensed by capacitive sensors embedded in the MA structure. An instrumented suspension is utilized for

\footnotetext{
This work was supported by the Information Storage Industry Consortium (INSIC) and the Computer Mechanics Laboratory (CML) of UC Berkeley.

X. Huang and R. Horowitz are with the Department of Mechanical Engineering, UC Berkeley, CA 94720 \{xhhuang, horowitz\} @me. berkeley.edu.

Y. Li is with the Maxtor Corporation, 500 McCarthy Blvd, Milpitas, CA 95035, yunfeng_1i@maxtor. com.
}

detection of airflow-excited suspension vibrations. The first controller is designed using the so-called PQ method [6], which explicitly considers the interference between the two actuators. Portions of the controller are designed sequentially by using frequency shaping techniques. The second controller is designed using a multi-objective optimization technique [7][8]. An $\mathrm{H}_{2}$ optimization problem is formulated with some $H_{\infty}$ bounds for robust stability. In both designs, the MA resonance mode is first damped using the relative motion signal from the MA, then a vibration control scheme is implemented using the strain signal from the instrumented suspension. For better performance, both the MA damping and vibration control are run at a higher rate than the basic servo loop. A a low rate track-following controller is then designed based on the downsampled damped plant.

This paper is organized as follows: Section 2 describes the modelling of the dual-stage actuator; Section 3 presents the two design methods: the PQ method and the multiobjective optimization using LMIs; Section 4 provides a detailed comparison between the two designed systems; Section 5 concludes this paper.

\section{Dual-Stage Model and InNer Loop Vibration CONTROL}

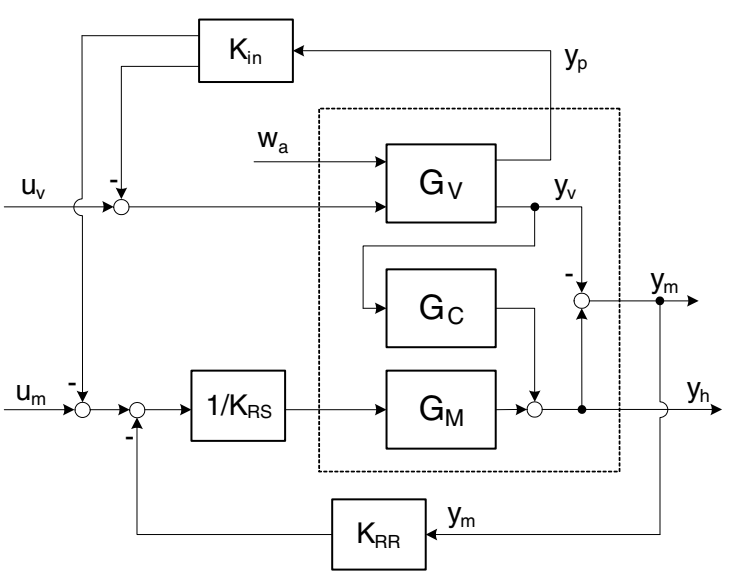

Fig. 1. Block diagram for MA damping and suspension vibration suppression.

The block diagram for the dual-stage actuator with an instrumented suspension is shown in the dashed frame in Fig. 1, and its frequency responses are shown in Fig. 2. In these figures, $u_{v}$ and $u_{m}$ are the control inputs to the VCM and MA respectively. $w_{a}$ denotes the airflow turbulence acting on the suspension and is exciting its vibration modes. 
$y_{h}, y_{p}, y_{m}$, and $y_{v}$ are the read/write head position, the strain sensor output, the relative motion of the MA, and the suspension tip position, respectively. In conventional singlestage disk drives, only $y_{h}$ is available in the form of the position error signal (PES). In dual-stage actuators with a MEMS MA and an instrumented suspension, $y_{p}$ and $y_{m}$ are measurable from the strain sensors on the suspension surface and the capacitive sensing embedded in the MA respectively. In all cases, $y_{v}$ is not measurable.

\section{A. Modelling of the Dual-stage Actuator}

In Fig. 1, $G_{V}$ denotes the dynamics of the VCMsuspension assembly and its transfer function can be written as

$$
G_{V}(s)=\frac{A_{0}}{s^{2}}+\sum_{i=1}^{6} \frac{A_{i}}{s^{2}+2 \zeta_{i} \omega_{i} s+\omega_{i}^{2}},
$$

where $\omega_{i}, \zeta_{i}$ and $A_{i}$ are the natural frequency, damping ratio and modal constant of mode $i$, respectively. The values of these modal parameters are determined by finite element analysis of a prototype suspension [9]. The first mode is the rigid body mode and cannot be detected by strain sensors. Six suspension modes are included in the model $G_{V}$, of which two major suspension modes, located at $7.4 \mathrm{kHz}$ and $10.7 \mathrm{kHz}$, will be considered in controller design, while the full-order model will be used for performance analysis of the designed closed-loop system.

$G_{M}$ denotes the dynamics of the MA. Its transfer function can be expressed as

$$
G_{\mathrm{M}}(s)=\frac{A_{m}}{s^{2}+2 \zeta_{m} \omega_{m} s+\omega_{m}^{2}} .
$$

This model is obtained based on the experimental results of a prototype MEMS MA fabricated in our research group. As can be seen, the MA has a single spring-mass mode with $\omega_{m}=2.2 \mathrm{kHz}$ and $\zeta_{m}=0.2 \mathrm{~N} \cdot \mathrm{s} / \mathrm{m}$. All other modes, such as the rotational mode, have been effectively suppressed by proper design and fabrication. A capacitive sensing structure has been embedded in the MA structure such that the motion of the MA relative to the suspension, $y_{m}=y_{h}-y_{v}$, can be measured.

Since the MA motion is translational, there is coupling between the two actuators, that is, VCM actuation can excite MA dynamics through the suspension tip. On the other hand, it may be reasonably assumed that MA actuation will not excite suspension dynamics due to the MA's small moving inertia. The coupling dynamics can be expressed as

$$
G_{\mathrm{C}}(s)=\frac{2 \zeta_{m} \omega_{m} s+\omega_{m}^{2}}{s^{2}+2 \zeta_{m} \omega_{m} s+\omega_{m}^{2}},
$$

with the same notation as in (2).

\section{B. Inner Loop Vibration Control}

Before designing a track-following controller, it is desirable to design some inner loop controllers to damp the MA mode using $y_{m}$, and to damp suspension modes using $y_{p}$. To this end, first discretize the dual-stage actuator at 50
$\mathrm{kHz}$, which implies that both $y_{m}$ and $y_{p}$ are sampled at 50 $\mathrm{kHz}$. The MA dynamics then become

$$
G_{\mathrm{MA}}\left(q^{-1}\right)=\frac{q^{-1} B_{o}\left(q^{-1}\right)}{A_{o}\left(q^{-1}\right)},
$$

where $q^{-1}$ is the one-step delay operator. The MA damping loop is illustrated in the lower part of Fig. 1. With the pole placement technique, the open-loop polynomial $A_{o}\left(q^{-1}\right)$ is replaced by the closed-loop polynomial $A_{R}\left(q^{-1}\right)$ by solving the following Diophantine equation with respect to $K_{R S}$ and $K_{R R}$ :

$$
A_{R}\left(q^{-1}\right)=A_{o}\left(q^{-1}\right) K_{R S}\left(q^{-1}\right)+q^{-1} B_{o}\left(q^{-1}\right) K_{R R}\left(q^{-1}\right) .
$$

Here $A_{R}\left(q^{-1}\right)$ is chosen by the designer, and usually the damping ratio is set to 1 .

After the MA is adequately damped, a suspension vibration damping and compensation controller $K_{\text {in }}$ is designed using $y_{p}$. The design of $K_{\text {in }}$ is formulated as an LQG problem. Consider the discrete-time representation of the plant that already incorporates MA damping:

$$
\begin{aligned}
x(k+1) & =A x(k)+B u(k)+B_{w} w_{a}(k) \\
y(k) & =C x(k)+n(k),
\end{aligned}
$$

where $y(k)=\left[y_{h}(k) y_{p}(k)\right]^{T}, u(k)=\left[u_{v}(k) u_{m}(k)\right]^{T}$, $w_{a}(k)$ and $n(k)$ are airflow turbulence and measurement noise respectively. The objective of $K_{\text {in }}$ is to minimize the following cost function

$$
J=E\left\{y_{h}^{2}(k)+R u^{2}(k)\right\},
$$

where $E$. is the expectation operator and $R$ is the control weighting matrix. The control action to the VCM takes effect by actively damping those suspension modes, while the control action to the MA takes effect by generating additional relative motion to compensate for the airflowexcited vibrations at the r/w head. Fig. 2 shows the control effect when the MA damping loop and the vibration damping and compensation loop are closed. The damped plant now becomes a double-input single-output (DISO) system.

\section{TRACK-FOLlOWING CONTROLlER DESIGN}

As mentioned in the previous section, the strain sensor output $y_{p}$ and the relative MA motion $y_{m}$ are sampled at $50 \mathrm{kHz}$, but the PES is available only at $25 \mathrm{kHz}$ due to hardware limitations. Thus the fast rate damped plant needs to be downsampled by a factor of 2 to accommodate the low rate PES. A low rate track-following controller can then be designed based on the low rate plant.

\section{A. $P Q$ Design}

The first design approach is called the PQ method [10], which reduces a control design problem for DISO systems to two standard control design problems for SISO systems. The first part of the PQ method directly addresses the issue of actuator output interference as a function of frequency, 


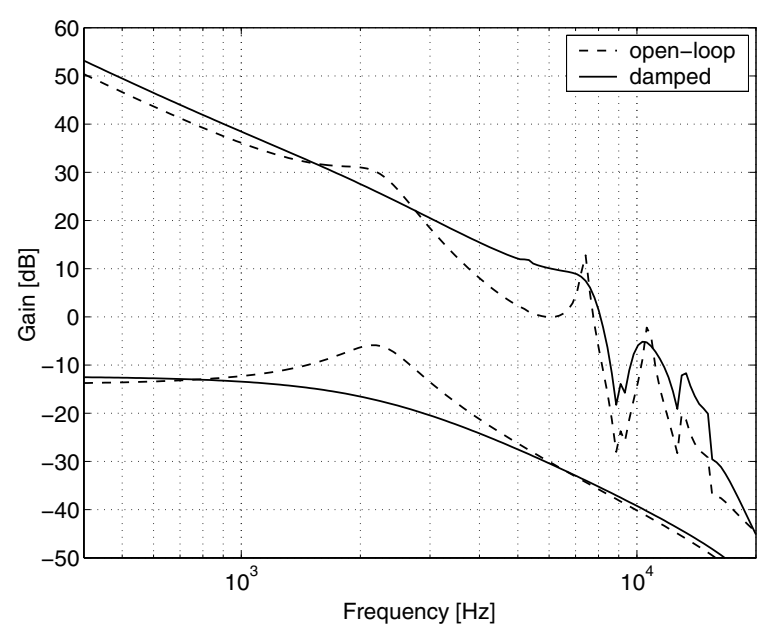

Fig. 2. Plant dynamics with/without vibration damping and compensation.

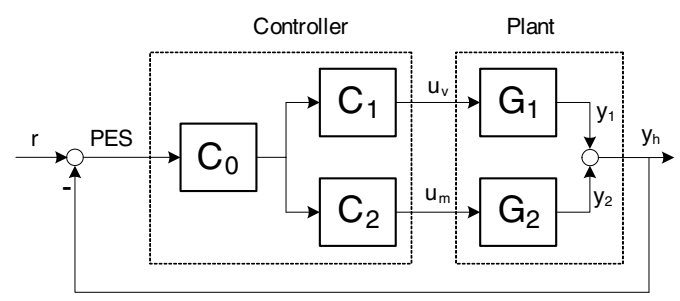

Fig. 3. Block diagram for the PQ design method.

and the second part allows the use of traditional loop shaping techniques to achieve the desired system performance. Its schematic is shown in Fig. 3, in which the plant is already closed by the MA damping and $K_{\text {in }}$.

To begin with, first define

$$
G_{\mathrm{P}}\left(e^{j \omega}\right):=\frac{G_{1}\left(e^{j \omega}\right)}{G_{2}\left(e^{j \omega}\right)},
$$

which is the ratio between the two input-output channels of the plant as shown in Fig. 3. Then design a compensator $G_{\mathrm{Q}}$ to stabilize the virtual plant $G_{\mathrm{P}}$ with unity feedback. The phase margin of the open-loop plant $G_{\mathrm{PQ}}:=G_{\mathrm{P}} G_{\mathrm{Q}}$ determines how much the outputs of the two actuators interfere cooperatively or destructively at the handoff frequency. A large phase margin is desired to ensure that the two actuators work constructively. The designed $G_{\mathrm{Q}}$ is then decomposed into two parts with $G_{\mathrm{Q}}=C_{1} / C_{2}$ such that both $C_{1}$ and $C_{2}$ are realizable.

Here, loop shaping techniques are used in designing $G_{\mathrm{Q}}$. The final $G_{\mathrm{Q}}$ includes a PI controller with break frequency at $100 \mathrm{~Hz}$ to achieve zero steady-state error, and a complexvalued lead compensator with peak phase of $20^{\circ}$ at $1.4 \mathrm{kHz}$ so that the phase margin of $G_{\mathrm{PQ}}$ is $56^{\circ}$. The frequency response of $G_{\mathrm{PQ}}$ is shown in Fig. 4. Since $G_{\mathrm{Q}}(z)$ is already realizable, it is straightforward to let $C_{2}(z)=1$ and $C_{1}(z)=G_{\mathrm{Q}}(z)$.

With $C_{1}$ and $C_{2}$ connected to $G_{1}$ and $G_{2}$ respectively, a

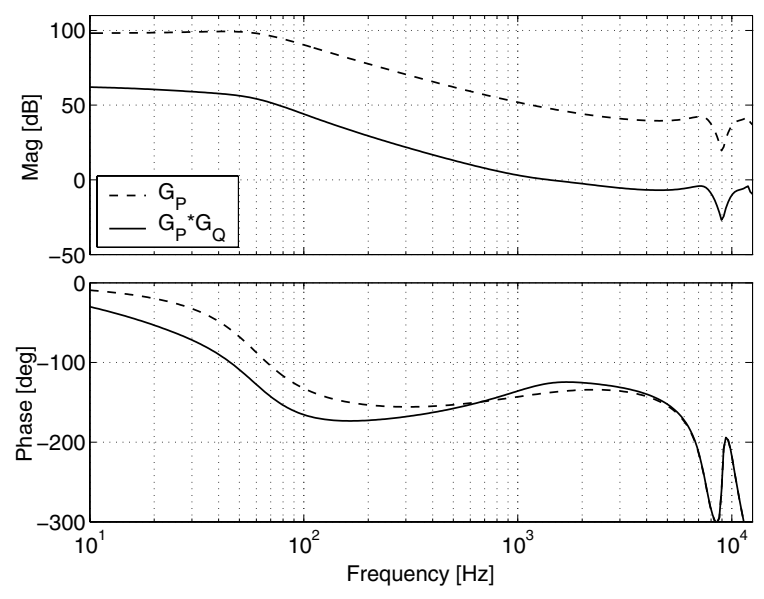

Fig. 4. Frequency responses of $G_{\mathrm{P}}$ and $G_{\mathrm{PQ}}$.

compensator $C_{0}$ is designed for the SISO plant

$$
G_{\text {siso }}:=C_{1} G_{1}+C_{2} G_{2} .
$$

This is again fulfilled by using loop shaping techniques. $C_{0}$ is designed to be a combination of a lag compensator $K_{\text {lag }}$ for better error rejection in the low frequency range, a lead compensator $K_{\text {lead }}$ for adequate phase margin around the crossover frequency, and a notch filter $K_{\text {notch }}$ for high frequency peak suppression. The open-loop frequency response of $G_{\text {siso }} C_{0}$ is shown in Fig. 5. The closed-loop system achieves a gain margin of $7.56 \mathrm{~dB}$ and a phase margin of $35.3^{\circ}$. The sensitivity transfer function is shown in Fig. 8 in Section IV.

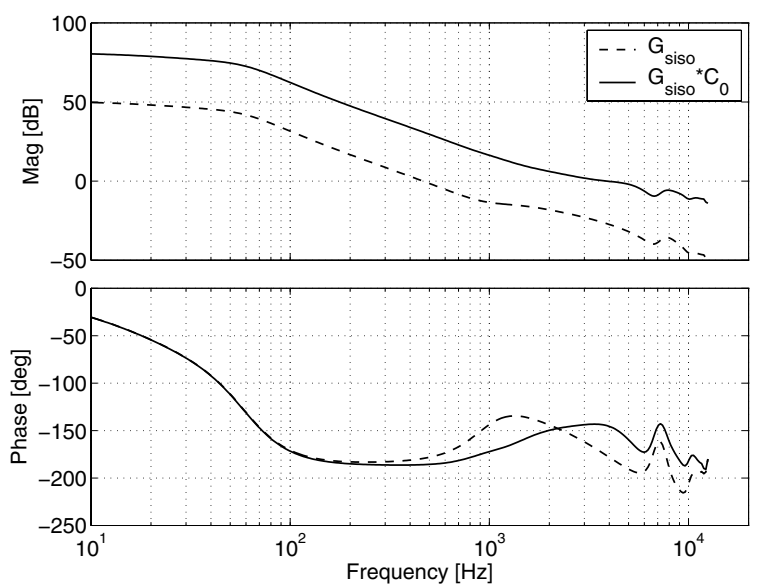

Fig. 5. Frequency responses of $G_{\text {siso }}$ and $G_{\text {siso }} C_{0}$.

\section{B. Multi-objective Optimization}

The second design approach for track-following control is based on a multi-objective optimization method [8]. This design method addresses two performance specifications simultaneously: the nominal track-following performance and stability robustness. Fig. 6 shows the schematic for this method. 


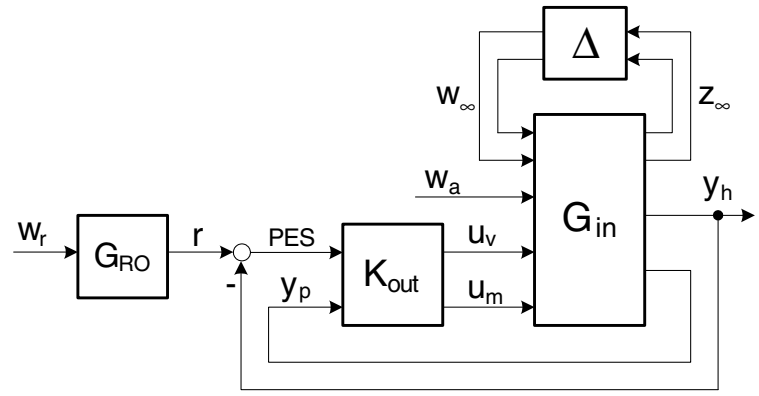

Fig. 6. Block diagram for multi-objective optimization method.

In the block diagram, $G_{\mathrm{RO}}$ is the frequency shaping function to generate the track runout from a normalized noise $w_{r} . G_{\text {in }}$ is the plant with the MA damping and $K_{\text {in }}$ closed, as shown in Fig. $1 . \Delta:=\operatorname{diag}\left[\Delta_{V}, \Delta_{M}\right]$ where $\Delta_{V}$ and $\Delta_{M}$ are multiplicative uncertainties of the nominal actuator model:

$$
\begin{aligned}
G_{V}(s) & =G_{V \text { nom }}(s)\left(1+\Delta_{V}(s) W_{V}(s)\right), \\
G_{M}(s) & =G_{M \text { nom }}(s)\left(1+\Delta_{M}(s) W_{M}(s)\right) .
\end{aligned}
$$

Here, $\left\|\Delta_{V}\right\|_{\infty} \leq 1,\left\|\Delta_{M}\right\|_{\infty} \leq 1$, and $W_{V}$ and $W_{M}$ are magnitude bounding functions of the two uncertainties.

The first design criterion is expressed as to minimize the RMS value of the PES:

$$
\min \operatorname{RMS}(\mathrm{PES}) \Leftrightarrow \min \left\|G_{z_{2} w_{2}}\right\|_{2},
$$

where $z_{2}=\left[\mathrm{PES}, u_{v}, u_{m}\right]^{T}$ and $w_{2}=\left[w_{r}, w_{a}\right]^{T}$.

Stability robustness of the closed-loop system is characterized by the structured singular value [11]:

$$
\sup _{\omega} \mu_{\Delta}\left(G_{\mathrm{cl}}(j \omega)\right)<1,
$$

where $G_{\mathrm{cl}}$ is the closed-loop plant. Since the dimension of $\Delta$ is only 2 , condition (12) can be approximated by the following two constraints $\left\|G_{z_{\infty} w_{\infty}, \Delta_{V}}\right\|_{\infty}<\gamma_{V}$ and $\left\|G_{z_{\infty} w_{\infty}, \Delta_{M}}\right\|_{\infty}<\gamma_{M}$, where $\gamma_{V}$ and $\gamma_{M}$ are selected by trial and error.

From the above discussion, we have shown that the tracking control design problem may be cast as an $\mathrm{H}_{2}$ norm minimization problem with some $H_{\infty}$ norm bounds, that is,

$$
\begin{array}{cl} 
& K_{\text {out }}=\arg \min _{K_{\text {out }}} \gamma_{2}, \\
\text { s.t. } & \left\|G_{z_{2} w_{2}}\right\|_{2}<\gamma_{2}, \\
& \left\|G_{z_{\infty} w_{\infty}, \Delta_{V}}\right\|_{\infty}<\gamma_{V}, \\
\text { and } & \left\|G_{z_{\infty} w_{\infty}, \Delta_{M}}\right\|_{\infty}<\gamma_{M} .
\end{array}
$$

This problem can be further represented as linear matrix inequalities (LMI) and be solved with some convex optimization algorithms [12]. The resulting full-order controller is reduced from order 21 to 14 without a noticeable performance degradation. The dynamics of the controller from the PES to $u_{v}$ are shown in Fig. 7. From the figure we see that when $H_{\infty}$ bounds are imposed, the controller gain in the high frequency range is reduced significantly to achieve greater stability margin. It is also noted that the difference between the full-order and reduced-order controllers is negligible.

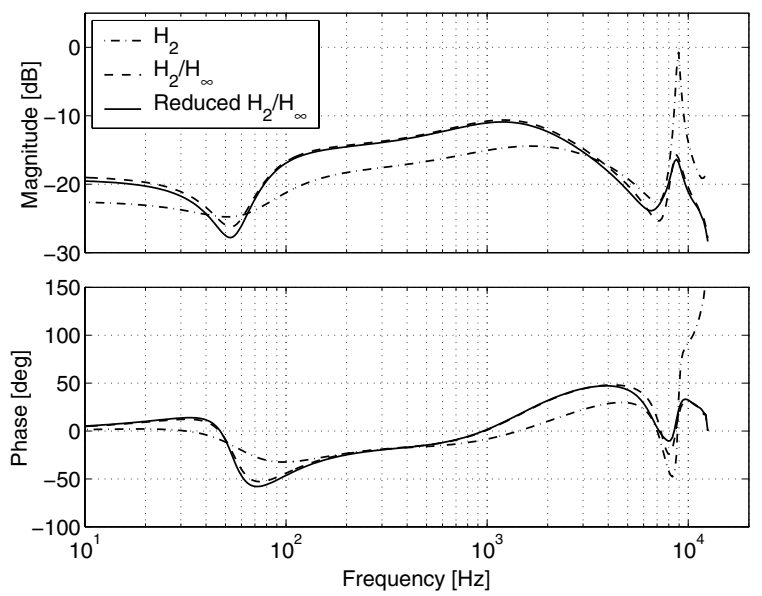

Fig. 7. Bode plots for various controllers. $H_{2}: H_{2}$ norm minimization only, which is equivalent to LQG design; $H_{2} / H_{\infty}$ : mixed-objective design with $H_{\infty}$ bounds; Reduced $H_{2} / H_{\infty}$ : reduced-order controller of the second one.

\section{Controller Synthesis Results}

\section{A. Frequency Response}

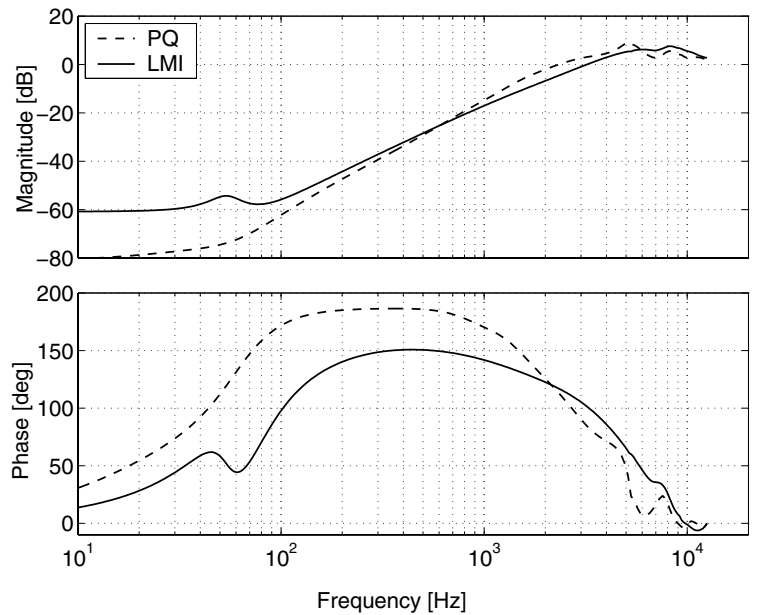

Fig. 8. Closed-loop sensitivity responses of the two designs.

Fig. 8 shows the closed-loop sensitivity responses of the two designs, where the multi-objective optimization method is abbreviated to the LMI method for clarity. From the figure, it is seen that the PQ design has greater error rejection in the low frequency range, and slightly larger amplification beyond $500 \mathrm{~Hz}$. Since the LMI design is a MIMO design method, its sensitivity characteristics have been optimally shaped in the sense of taking into consideration all those modelled disturbances and guaranteeing robust stability. Also from the figure we see that the closed-loop servo bandwidth for the PQ design and the LMI design are 2.1 $\mathrm{kHz}$ and $3 \mathrm{kHz}$, respectively. 


\section{B. Stability Robustness}

Modelling of parametric uncertainties usually yields a high-dimensional, highly structured $\Delta$ block whose stability condition can be more precisely characterized by the structured singular value $\mu$ rather than its $H_{\infty}$ norm [11]. In this analysis, parameter perturbation in the full-order plant is assumed as follows. The resonance frequency of each mode of the VCM has a $\pm 8 \%$ variation, and that of the MA mode has a $\pm 12 \%$ variation. All damping factors have a $\pm 20 \%$ variation, and all modal constants have a $\pm 5 \%$ variation. All these parameter uncertain ranges have been normalized. In addition, the multirate sampling property of the system is taken into account by lifting the high rate damped plant before connecting to the low rate outer loop controller, as shown in Fig. 9. Multiplicity of each uncertain parameter is therefore doubled after this operation. The final $\mu$ plot for the two designs are shown in Fig. 10.

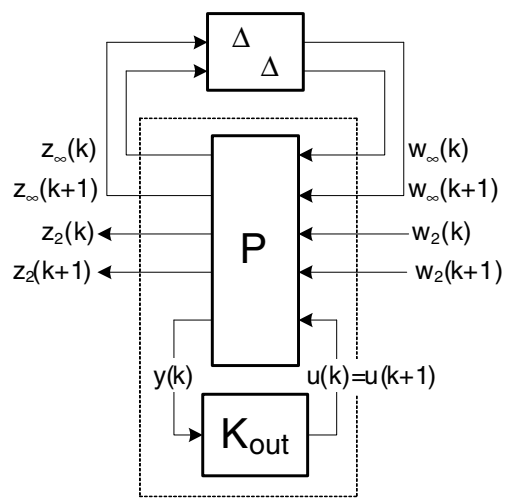

Fig. 9. Plant lifted to retain the multirate sampling property.

Some similarities can be observed between the two curves. The peaks around $2 \mathrm{kHz}$ are attributed to variation of the MA mode. The peaks around $5 \mathrm{kHz}$ correspond to the open-loop crossover frequencies of the two systems, which are usually characterized by gain and phase margins. Two large peaks around $10 \mathrm{kHz}$ and $12 \mathrm{kHz}$ result from the undamped modes around $15 \mathrm{kHz}$ and $13 \mathrm{kHz}$, respectively, in which the first mode has been aliased when lifting the high-rate plant. The two peaks around $7.4 \mathrm{kHz}$ and $10.2 \mathrm{kHz}$ are small due to the damping effect of the instrumented suspension. The maximum $\mu$ values are 0.8 for the PQ design and 0.93 for the LMI design, implying by $\mu$ theory that both of the two systems are robustly stable with respect to the assumed parameter variations.

\section{Performance Robustness}

Although stability of the closed-loop systems is guaranteed over certain variations of modal frequencies and damping coefficients, the system performance may degrade to become unacceptable before the stability condition is violated. This section investigates the performance robustness to plant variations by analysis and simulation of the two systems.

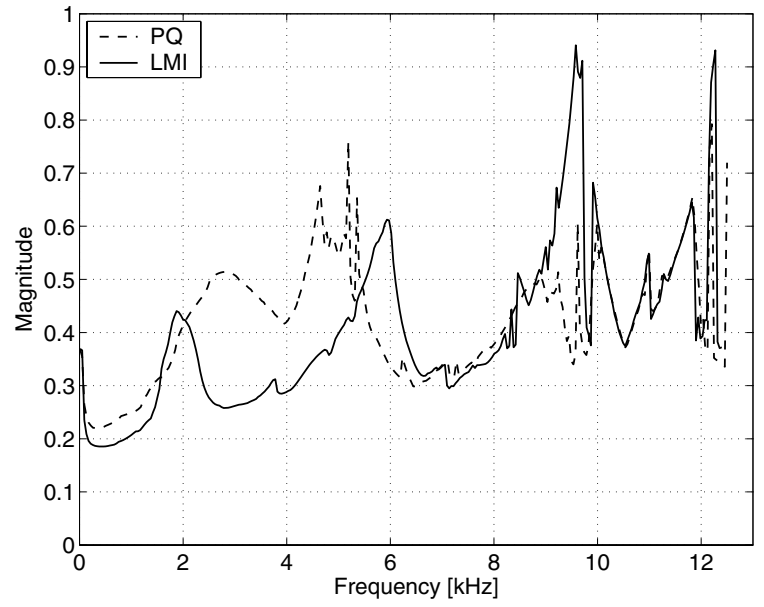

Fig. 10. $\mu$ plots for parametric uncertainties of the two designs.

1) Worst-case Performance Estimation: The estimation of the worst-case performance, or robust performance, of the two systems can be conducted by solving additional LMIs. For parametric uncertainties, the $\Delta$ block has the following structure

$$
\boldsymbol{\Delta}:=\left\{\operatorname{diag}\left[\delta_{1} I_{r_{1}}, \cdots, \delta_{N_{p}} I_{r_{N_{p}}}\right]: \delta_{i} \in \mathbb{R},\left|\delta_{i}\right| \leq 1\right\} .
$$

in which each parameter variation $\delta_{i}$ is repeated $r_{i}$ times. If $\Delta$ is LTI, the uncertain system $(T, \Delta)$ is said to have a robust $H_{2}$ performance level $\gamma$ if it is robustly stable and

$$
\sup _{\Delta \in \Delta}\left\|T_{z_{2} w_{2}}(\Delta)\right\|_{2}<\gamma .
$$

To estimate $\gamma$, define the following sets

$$
\begin{aligned}
\boldsymbol{X} & :=\left\{\operatorname{diag}\left[X_{1}, \cdots, X_{N_{p}}\right]: X_{i} \in \mathbb{C}^{r_{i} \times r_{i}}, X_{i}>0\right\}, \\
\boldsymbol{G} & :=\left\{\operatorname{diag}\left[G_{1}, \cdots, G_{N_{p}}\right]: G_{i}=G_{i}^{*} \in \mathbb{C}^{r_{i} \times r_{i}}\right\}, \\
\boldsymbol{Y} & :=\left\{Y=Y^{*} \in \mathbb{C}^{N_{w_{2}} \times N_{w_{2}}}\right\} .
\end{aligned}
$$

Suppose there exists $X(\omega) \in \boldsymbol{X}, G(\omega) \in \boldsymbol{G}$, and $Y(\omega) \in$ $\boldsymbol{Y}$, such that

$$
\begin{aligned}
& j\left(\left[\begin{array}{cc}
G(\omega) & 0 \\
0 & 0
\end{array}\right] T(\omega)-T^{*}(\omega)\left[\begin{array}{cc}
G(\omega) & 0 \\
0 & 0
\end{array}\right]\right) \\
+ & T^{*}(\omega)\left[\begin{array}{cc}
X(\omega) & 0 \\
0 & I
\end{array}\right] T(\omega)-\left[\begin{array}{cc}
X(\omega) & 0 \\
0 & Y(\omega)
\end{array}\right]<0
\end{aligned}
$$

holds for all $\omega \in[02 \pi]$, and

$$
\int_{0}^{2 \pi} \operatorname{trace}(Y(\omega)) \frac{d \omega}{2 \pi}<\gamma^{2},
$$

then the system $(T, \Delta)$ is robustly stable and has a robust performance level $\gamma$ [13].

Since this is a convex optimization problem, it can readily be solved through use of LMIs. The estimated results of $\operatorname{trace}(Y(\omega))$ are shown in Figs. 11 and 12. The nominal $H_{2}$ performance $(\Delta=0)$ is also shown for comparison. The ratio between $\gamma_{\text {robust }}$ and $\gamma_{\text {nom }}$ is 1.59 for the PQ design and 1.35 for the LMI design, indicating better performance 
robustness for the LMI design. Also note that the major portions of the tracking error are located around $2 \mathrm{kHz}$ and $5 \mathrm{kHz}$, which are consistent with the results shown in Fig. 10: performance degradation mainly results from MA mode variation and decreased gain/phase margins.

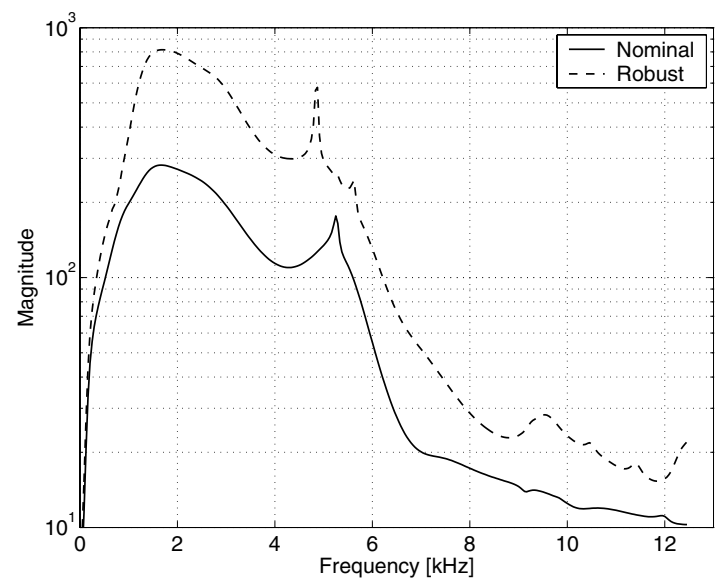

Fig. 11. Robust performance of the PQ design.

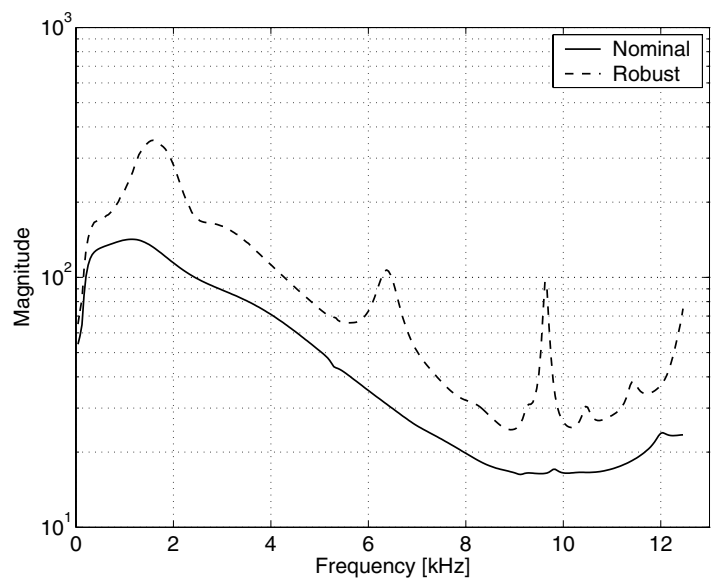

Fig. 12. Robust performance of the LMI design.

TABLE I

COMPARISON OF ROBUST PERFORMANCE

\begin{tabular}{|c|c|c|c|}
\hline & \multicolumn{3}{|c|}{ RMS of PES [nm] } \\
\hline Design method & min & nominal & $\max$ \\
\hline PQ & 5.21 & 4.80 & 4.87 \\
\hline LMI & 3.88 & 3.76 & 3.93 \\
\hline
\end{tabular}

2) Time-domain Simulation: Finally, time-domain simulations are conducted to verify the above results. As during design, the sampling frequency of the PES is $25 \mathrm{kHz}$, and $y_{p}$ and $y_{m}$ are sampled at $50 \mathrm{kHz}$. Track runout, airflow disturbance and measurement noises are injected into the plant at corresponding locations. Table I shows the RMS values of PES of the two systems with parameter variations.
Here, "nominal" denotes the nominal plant, and "min" ("max") denotes the plant in which each parameter takes on the smallest (largest) value within its uncertainty range. From this table, it can be seen that the LMI design is better than the PQ design in not only nominal performance, $3.76 \mathrm{~nm}$ vs. $4.80 \mathrm{~nm}$, but also performance robustness, $4.5 \%$ vs. $8.5 \%$ in performance degradation, exhibiting good consistency with the results shown in Figs. 11 and 12.

\section{CONCLUSION}

In this paper, two track-following controllers were designed and analyzed on a dual-stage system with an instrumented suspension. The first controller is designed using the PQ method and the second uses a multi-objective optimization technique using LMIs. With explicit consideration of plant uncertainty in the design process, the LMI design can achieve better robust stability and robust performance. However, it is noted that even after applying order reduction, the LMI method still yields a controller with higher degree than that of the PQ method, which may introduce some deterioration in performance. These design results will be experimentally verified on an integrated hardware system that will soon be available in our research group.

\section{REFERENCES}

[1] Y. Li and R. Horowitz, "Active vibration control of a pzt actuated suspension in hard disk drives," in Proc. Amer. Control Conf., Anchorage, AK, May 2002, pp. 1366-1371.

[2] M. Kobayashi, S. Nakagawa, and S. Nakamura, "A phase-stablized servo controller for dual-stage actuators in hard disk drives," IEEE Trans. Magnetics, vol. 39, no. 2, pp. 844-850, 2003.

[3] L.-S. Fan, R. Hirano, J. Hong, P. R. Webb, W. H. Juan, W. Y. Lee, S. Chan, T. Semba, W. Imaino, T. S. Pan, S. Pattanaik, F. C. Lee, I. McFadyen, S. Arya, and R. Wood, "Electorstatic microactuator and design considerations for HDD application," IEEE Trans. Magnetics, vol. 35, no. 2, pp. 1000-1005, March 1999.

[4] D. Horsley, N. Wongkomet, R. Horowitz, and A. Pisano, "Precision positioning using a microfabricated electrostatic actuator," IEEE Trans. Magnetics, vol. 35, no. 2, pp. 993-999, March 1999.

[5] H. Toshiyoshi, "Electrostatic microactuators for hdd piggyback systems," http://toshi.fujita3.iis.u-tokyo.ac.jp, 2002.

[6] W. Messner and R. Ehrlich, "A tutorial on controls for disk drives," in Proc. Amer. Control Conf., Arlington, VA, June 2001, pp. 408-420.

[7] M. C. D. Oliveira, J. C. Geromel, and J. Bernussou, "Extended $\mathrm{H}_{2}$ and $H_{\infty}$ norm characterizations and controller parametrizations for discrete-time systems," Int. J. Control, vol. 75, no. 9, pp. 666-679, 2002.

[8] X. Huang, R. Nagamune, R. Horowitz, and Y. Li, "Design and analysis of a dual-stage disk drive servo system using an instrumented suspension," in Proc. Amer. Control Conf., Boston, MA, June 2004, pp. $535-540$.

[9] K. Oldham, S. Kon, and R. Horowitz, "Fabrication and optimal strain sensor placement in an instrumented disk drive suspension for vibration suppression," in Proc. Amer. Control Conf., Boston, MA, June 2004, pp. 1855-1860.

[10] S. J. Schroeck and W. C. Messner, "On controller design for linear time-invariant dual-input single-output systems," in Proc. Amer. Control Conf., San Diego, CA, June 1999, pp. 4122-4126.

[11] G. J. Balas, J. C. Doyle, K. Glover, A. Packard, and R. Smith, $\mu-$ Analysis and Synthesis Toolbox for use with MATLAB. MUSYN Inc. and The MathWorks, Inc., USA, 1995.

[12] J. F. Sturm, Using Sedumi 1.05, A MATLAB Toolbox for Optimization over Symmetric Cones, 2001.

[13] F. Paganini, "Frequency domain conditions for robust $H_{2}$ performance," IEEE Trans. Automat. Control, vol. 44, no. 1, pp. 95-103, Jan 1999. 